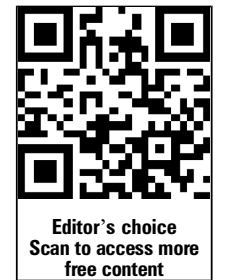

- Additional material is published online only. To view please visit the journal online (http://dx.doi.org/10.1136/ emermed-2012-201900).

${ }^{1}$ Division of Emergency Medicine, Children's National Medical Center, George Washington University, Washington, DC, USA ${ }^{2}$ Division of Pediatric Emergency Medicine, Department of Emergency Medicine, New York University School of Medicine, New York New York, USA

${ }^{3}$ Department of Emergency Medicine, University of Botswana School of Medicine Gaborone, Botswana ${ }^{4}$ Department of Emergency Medicine, University of British Columbia and St. Paul's Hospital, Vancouver, British Columbia, Canada

Correspondence to Dr Paul C Mullan, Division of Emergency Medicine, 111 Michigan Ave NW, Washington DC 20010, USA.

mullan20@gmail.com

Received 25 August 2012 Revised 16 January 2013 Accepted 20 January 2013 Published Online First 13 February 2013
To cite: Mullan $P C$, Torrey SB, Chandra A, et al. Emerg Med J 2014;31: 356-360.

\title{
Reduced overtriage and undertriage with a new triage system in an urban accident and emergency department in Botswana: a cohort study
}

\author{
Paul C Mullan, ${ }_{1}^{1}$ Susan B Torrey, ${ }^{2}$ Amit Chandra, ${ }^{3}$ Ngaire Caruso, ${ }^{3}$ Andrew Kestler ${ }^{4}$
}

\begin{abstract}
Background Improvements in triage have demonstrated improved clinical outcomes in resourcelimited settings. In 2009, the Accident and Emergency (A\&E) Department at the Princess Marina Hospital (PMH) in Botswana identified the need for a more objective triage system and adapted the South African Triage Scale to create the PMH A\&E Triage Scale (PATS).

Aim The primary purpose was to compare the undertriage and overtriage rates in the PATS and pre-PATS study periods.

Methods Data were collected from 5 April 2010 to 1 May 2011 for the PATS and compared with a database of patients triaged from 1 October 2009 to 24 March 2010 for the pre-PATS. Data included patient disposition outcomes, demographics and triage level assignments.
\end{abstract}

Results 14706 (pre-PATS) and 25243 (PATS) patient visits were reviewed. Overall, overtriage rates improved from $53 \%$ (pre-PATS) to $38 \%$ (PATS) $(p<0.001)$; likewise, undertriage rates improved from $47 \%$ (prePATS) to $16 \%$ (PATS) $(p<0.001)$. Statistically significant decreases in both rates were found when paediatric and adult cases were analysed separately. PATS was more predictive of inpatient admission, Intensive Care Unit (ICU) admission and death rates in the A\&E than was the pre-PATS. The lowest acuity category of each system had a $0.6 \%$ (pre-PATS) and 0\% (PATS) chance of death in the A\&E or ICU admission ( $p<0.001)$. No change in death rate was seen between the pre-PATS and PATS, but ICU admission rates decreased from $0.35 \%$ to $0.06 \%(p<0.001)$.

Conclusions PATS is a more predictive triage system than pre-PATS as evidenced by improved overtriage, undertriage and patient severity predictability across triage levels.

\section{INTRODUCTION}

Despite the benefits of effective triage and the consensus by emergency medicine (EM) experts of its importance, ${ }^{12}$ it is often performed poorly, especially in resource-limited settings. ${ }^{3}$ However, several triage systems have been developed and demonstrated positive outcomes in resource-limited settings. ${ }^{4}$

\section{Background in Botswana}

The Princess Marina Hospital (PMH) is a 533-bed facility in Gaborone, Botswana. The Accident and Emergency (A\&E) Department at $\mathrm{PMH}$ handles the acute care needs for southern Botswana. In 2009, there was no formal training in triage in the PMH A\&E. Triage nurses categorised patients subjectively as 'I' (life-threatening), 'II' (potentially life- threatening) or 'III' (non-life-threatening). In August 2009, the concept of improving the triage system was introduced and met with enthusiasm from the A\&E staff.

The project implementation team chose the South African Triage Scale (SATS) ${ }^{5}{ }^{6}$ as the model for developing a new triage system because they considered that South Africa had relatively similar patient presentations and resource constraints. The SATS 4-level system includes red ('immediate care'), orange ('very urgent'), yellow ('urgent') and green ('routine') categories that determine the order patients will be seen by the physician. ${ }^{7}$ A blue ('dead on arrival with no resuscitation') category is also designated for patients who need death certifications. The SATS uses age-appropriate vital sign parameters (heart rate, respiratory rate, temperature for all patients and additionally blood pressure for adults), mobility, mental status and presence of trauma to create a Triage Early Warning Score ${ }^{6}$ that places the patient into one of the four levels. The presence of certain clinical discriminators (eg, chest pain, stridor) can upgrade a patient into a more acute triage category. Last, a senior healthcare professional can override the system to determine a triage category as needed. The SATS has been in use since 2006 and has prospectively demonstrated low undertriage and overtriage rates and reduced patient waiting times. ${ }^{5} 8$

The team made minor modifications to the SATS using a modified Delphi process to make it clinically and culturally relevant in Botswana. The scale was then renamed the PMH A\&E Triage Scale (PATS; see online supplementary files 1-4). A\&E physicians, nurses and nurse assistants were trained to use PATS. ${ }^{3}$ PATS was implemented on 5 April 2010 and replaced pre-PATS.

The primary outcome of this study was to compare overtriage and undertriage rates of patients before and after PATS implementation. Overtriage occurs when a patient receives a high acuity triage assignment (red or yellow) and is subsequently discharged. Undertriage occurs when a patient receives a low acuity assignment (green) and subsequently dies or is admitted. We hypothesised that PATS would lead to significant improvements in our primary outcomes.

\section{METHODS}

\section{Study location}

$\mathrm{PMH}$ is the tertiary referral government hospital in Botswana and has an A\&E annual volume of approximately 30000 patients. In April 2010, the PMH A\&E staff included two EM specialists, 
Table 1 Pre-PATS and PATS subjects

\begin{tabular}{lclr}
\hline & $\begin{array}{l}\text { Pre-PATS period } \\
\% \text { per category }(\mathbf{n})\end{array}$ & $\begin{array}{l}\text { PATS period } \\
\% \text { per category }(\mathbf{n})\end{array}$ & $\mathbf{p}$ Value \\
\hline Gender: Female & $54 \%(8004)$ & $54 \%(13557)$ & 0.16 \\
Age 0-1.9 months & $1.3 \%(197)$ & $1.9 \%(491)$ & $<0.05$ \\
Age 2 months-12 years & $15 \%(2145)$ & $17 \%(4180)$ & $<0.05$ \\
Age 13-64 years & $73 \%(10715)$ & $75 \%(18817)$ & $<0.05$ \\
Age 65 years and older & $6 \%(821)$ & $7 \%(1655)$ & $<0.05$ \\
Specific age unrecorded & $6 \%(828)$ & $0.4 \%(100)$ & $<0.05$ \\
Total patients & 14706 & 25243 & \\
\hline
\end{tabular}

PATS, Princess Marina Hospital Accident and Emergency Triage Scale.

12 medical officer physicians (general practitioners assigned to $\mathrm{A} \& \mathrm{E}), 44$ nurses and five healthcare auxiliaries. The department had a triage room, two resuscitation beds, 10 curtained patient bays, one isolation room and seven hallway beds. Triage was typically performed by one nurse in the triage room after which triaged patients were sent to the waiting room or directly to an A\&E bed.

\section{Data collection}

Patient information in the A\&E was recorded on a single government issued duplicate form that captured triage information, physician documentation, orders and disposition. The team modified the pre-PATS triage form to capture PATS-specific information. One copy was kept for A\&E record keeping, and the other remained with the patient's file. Information was entered into a computer database (Microsoft Excel 2007; after July 2010, Microsoft Access 2010) by healthcare auxiliaries who periodically underwent quality assurance evaluation by EM specialists to ensure accuracy.

\section{Study design and subjects}

This retrospective observational cohort study included a pre-PATS period (1 October 2009 to 28 March 2010) and PATS period (5 April 2010 to 1 May 2011). Between the study periods was a week-long washout period during which the PATS training for A\&E staff and a national holiday occurred. Patients were eligible for inclusion if they were seen in the A\&E during either study period and had a triage form completed that was entered into the database. All ages were included. Paediatric age was defined as $\leq 12$ years and adult age as $\geq 13$ years.

For our primary outcome, overtriage and undertriage rates for the two periods were compared using equivalent definitions to the SATS validation study. ${ }^{6}$ Overtriaged patients were those who received triage categories of pre-PATS-I, PATS-red, or PATS-orange and were discharged from the A\&E. Undertriaged patients were those with pre-PATS-III or PATS-green who were admitted to the hospital or died in the A\&E. Subjects were excluded from primary outcome analysis if they had no triage assignment, no final disposition or were dead on arrival.

For our secondary outcomes, we examined the following parameters before and after implementation of PATS: distribution of triage categories, percentage of patients without assigned triage categories, percentage of each triage category with 'hospitalised patients' (defined as admitted to the ward or Intensive Care Unit (ICU), or died in the A\&E), ${ }^{9}$ proportion of 'critical patients' (defined as ICU admission or died in A\&E) in each triage category and percentage of critical outcomes in the lowest triage categories.

\section{Data analysis and outcomes}

Statistical analyses were performed using STATA V.11.1 (College Station, Texas, USA) and Microsoft Excel 2007 (Seattle, Washington, USA). Two sample tests of proportion were used to compare the two samples; a $\mathrm{p}$ value of $<0.05$ was considered statistically significant.

Given the reported SATS overtriage rate of $45 \%,{ }^{6}$ we considered an absolute difference of $5 \%$ in overtriage rate between the pre-PATS and PATS study periods to be a significant change for our primary outcome. We calculated a minimum sample size of 1204 pre-PATS and 2408 PATS visits $(\alpha=0.05, \beta=0.20$, ratio of pre-SATS to SATS patients of 1:2).

Graphical displays ${ }^{3}$ were constructed to compare the distribution of triage levels and the proportion of various outcomes (death in the A\&E, ICU admission, hospitalised and critical patients) across categories for each triage system; these were used because we would be comparing two triage systems that had a different number of levels (three for pre-PATS, four for PATS). The proportions of hospitalised patients in the most and least severe triage categories of each system were also compared.

\section{Ethical considerations and conflicts of interest}

Protected health information was removed from the database prior to analysis to preserve patient confidentiality. The study received institutional review board approvals at the Botswana Ministry of Health, University of Botswana, PMH and Baylor College of Medicine. Subjects incurred no costs based on their involvement in this project.

\section{RESULTS}

In the two study periods, 14706 (pre-PATS) and 25243 (PATS) patients were included for analysis (table 1). For our primary

Table 2 Final dispositions of patients in pre-PATS and PATS study periods

\begin{tabular}{llll}
\hline Disposition & $\begin{array}{l}\text { Pre-PATS period } \\
\%(95 \% \text { Cl) number }\end{array}$ & $\begin{array}{l}\text { PATS period } \\
\%(95 \% \text { Cl) number }\end{array}$ & p Value \\
\hline Admitted to ward or surgery & $41.9(41.1$ to 42.7$) 6161$ & $43.7(43.1$ to 44.3$) 11038$ \\
Discharge to home or clinic & $43.8(42.6$ to 45.0$) 6446$ & $49.6(49$ to 50.2$) 12523$ & $<0.05$ \\
Dead on arrival & $1.56(1.35$ to 1.75$) 228$ & $1.64(1.48$ to 1.80$) 414$ & 0.05 \\
Left without being seen & $0.01(0.01$ to 0.03$) 2$ & $0.48(0.39$ to 0.56$) 120$ & 0.49 \\
Died in A\&E & $0.19(0.12$ to 0.26$) 28$ & $0.19(0.13$ to 0.24$) 47$ & 0.05 \\
Admitted to ICU & $0.35(0.25$ to 0.44$) 51$ & $0.06(0.03$ to 0.09$) 16$ & $<.93$ \\
Unknown final disposition & $12.2(10.7$ to 13.7$) 1790$ & $4.3(4.1$ to 4.6$) 1085$ & $<0.05$ \\
\hline ICU, Intensive Care Unit; PATS, Princess Marina Hospital Accident and Emergency Triage Scale. & &
\end{tabular}


Table 3 Undertriage and overtriage rates by age

\begin{tabular}{lllc}
\hline & $\begin{array}{l}\text { Pre-PATS period } \\
(95 \% \mathrm{Cl})\end{array}$ & $\begin{array}{l}\text { PATS period } \\
(95 \% \mathrm{Cl})\end{array}$ & p Value \\
\hline Overtriage: all ages & $52.5(45.6$ to 59.4$)$ & $38.4(37.5$ to 39.3$)$ & $<0.001$ \\
Overtriage: adults & $57.1(48.9$ to 65.3$)$ & $41.4(40.3$ to 42.5$)$ & $<0.001$ \\
Overtriage: paediatric & $42.9(29.0$ to 56.7$)$ & $28.8(27.1$ to 30.6$)$ & 0.03 \\
Undertriage: all ages & $46.9(45.4$ to 48.3$)$ & $16.0(14.6$ to 17.4$)$ & $<0.001$ \\
Undertriage: adults & $45.9(44.3$ to 47.4$)$ & $14.9(13.4$ to 16.5$)$ & $<0.001$ \\
Undertriage: paediatric & $51.7(47.8$ to 55.7$)$ & $21.9(17.8$ to 26.0$)$ & $<0.001$ \\
\hline \multicolumn{2}{l}{ PATS, Princess Marina Hospital Accident and Emergency Triage Scale. } &
\end{tabular}

outcome, 2023 pre-PATS and 1933 PATS patients were excluded leaving 12683 pre-PATS and 23310 PATS patients for analysis; exclusion reasons included no triage assignment (five pre-PATS, 480 PATS patients), no final disposition (1790 pre-PATS, 1139 PATS patients) and dead on arrival status (228 pre-PATS, 414 PATS patients). Patient data were available for all days during the pre-PATS study period. There were 8 days in the PATS study period for which there were no data. The pre-PATS and PATS study periods averaged 82 and 64 recorded patients per day, respectively. Of these pre-PATS and PATS patients, the majority were within the 13- to 64-year-old category. Although there were statistically significant differences in the proportions of various age groups, the maximum difference was $2 \%$, and there were significantly more patients without a recorded age in the pre-PATS $(6 \%)$ than in the PATS $(0.4 \%)$ study period.

The percentages of patients with each final disposition in the pre-PATS and PATS study periods are described in table 2 . Overtriage and undertriage improved significantly $(\mathrm{p}<0.001)$ for all ages combined in the PATS study period (table 3). Significant decreases were also observed for overtriage and undertriage rates in the PATS study period when analyses were divided into adult and paediatric age groups.

The distribution of triage categories for the pre-PATS and PATS study periods is displayed graphically in figure 1 . The rate of hospitalised patients within each category is depicted in figure 2. In the PATS study period, there were increasing percentages of critical patients in the higher triage categories, with a significantly higher proportion of hospitalised patients in the highest (red) than in the lowest (green) categories $(p<0.001)$. In the pre-PATS period, the proportion of hospitalised patients in the highest (I) and lowest (III) triage categories were not significantly different $(\mathrm{p}=0.86)$.

In table 2 , the rate of death in the $A \& E$ did not change between the study periods $(p=0.93)$, but the rate of ICU admission did decrease significantly in the PATS study period $(p<0.001)$. Most of the critical patients in the pre-PATS period were assigned a level II triage category (figure 3), and more than a third of these patients received a level III designation ('not lifethreatening'). In the PATS period, the majority (75\%) of these critical patients were in the red category. For the lowest triage categories, the percentage of critical patients was significantly higher with pre-PATS level III $(0.6 \%)$ than in PATS level green patients $(0 \%)(\mathrm{p}<0.001)$.

\section{DISCUSSION}

The PATS generated a triage system that was more predictive of patient acuity than the pre-PATS in the A\&E at PMH. Overtriage was reduced significantly. As a result, providers could focus resources on a smaller, sicker group of patients. Decrease in undertriage rates with PATS assured that care for sicker patients was not delayed because they were inappropriately assigned lower triage categories. The PATS-green patients also had a much lower risk of having a critical outcome than did the pre-PATS-III patients, suggesting that the PATS is potentially a safer system for those who are triaged into the lowest category.

The outcomes for SATS and PATS implementation were similar, suggesting that they are reliable and valid triage tools in the A\&E settings in Southern Africa. Neither the SATS overtriage rates $(45 \%(95 \%$ CI $38 \%$ to $52 \%))$ nor undertriage rates (12\% (95\% CI 7\% to 17\%)) were significantly different from PATS. ${ }^{6}$ There were several differences between the studies, however. For SATS, triage was performed independently by an EM physician and nurse on weekdays between 08:00 and 17:00. Our PATS sample covered all times of the week, and we did not have an emergency specialist observing nurses triage every patient.

Trying to design a triage system that maximises specificity (undertriage) to avoid delaying care for sick people while maximising sensitivity (overtriage) to avoid wasting resources is a challenge for any setting. Attempts to decrease undertriage with further system modifications often leads to tradeoff increases in overtriage. ${ }^{10}$ The American College of Surgeons Committee on Trauma standards aim for EM overtriage and undertriage rates of less than $50 \%$ and $10 \%$, respectively, for trauma patients. ${ }^{11}$ The Emergency Severity Index (ESI), ${ }^{12}$ a five-level triage system used in the USA and validated on adult EM patients, showed hospitalisation rates in their four highest categories $(92 \%, 61 \%$, $26 \%$ and $10 \%$ ) that were not statistically significantly different from our red, orange, yellow and green hospitalisation rates (all p >0.05). ${ }^{9}$

There were no differences between the study periods in death rates within the A\&E. This may be because the overall rates were quite low. The significant decrease in the proportion of ICU admissions after PATS implementation may reflect more timely stabilisation and treatment in the A\&E. There was a higher rate of PATS patients who did not have a recorded triage scale assignment $(1.9 \%$ vs $0.03 \%)$. It is possible that some of
Figure 1 Proportion of patients assigned to each triage category in the pre-PATS and PATS study periods. (A) Distribution of triage categories during the pre-PATS study period. Unk = Unrecorded triage level. (B) Distribution of triage categories during the PATS study period. Unk = Unrecorded triage level. PATS, Princess Marina Hospital Accident and Emergency Triage Scale.
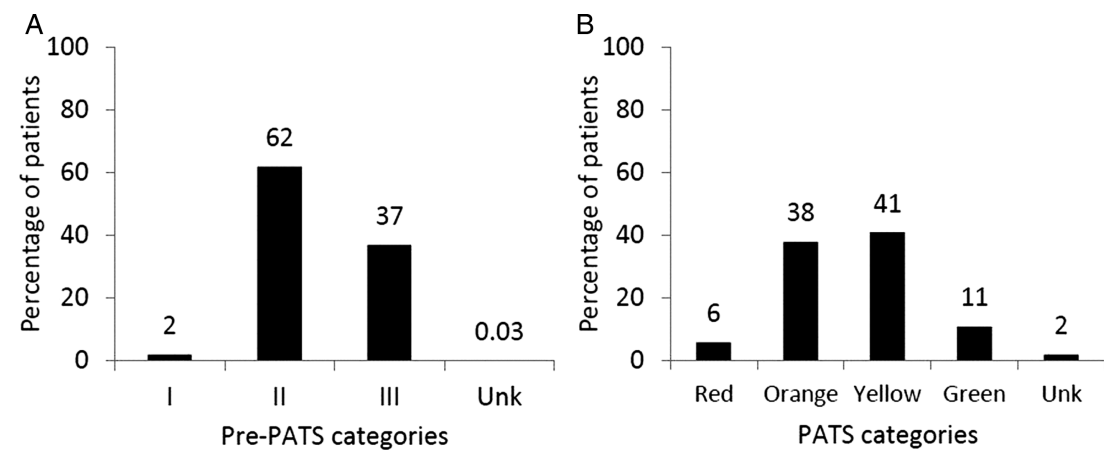
Figure 2 Proportion of each triage category that were hospitalised patients in the study periods. (A) Pre-PATS study period. (B) PATS study period. PATS, Princess Marina Hospital Accident and Emergency Triage Scale.
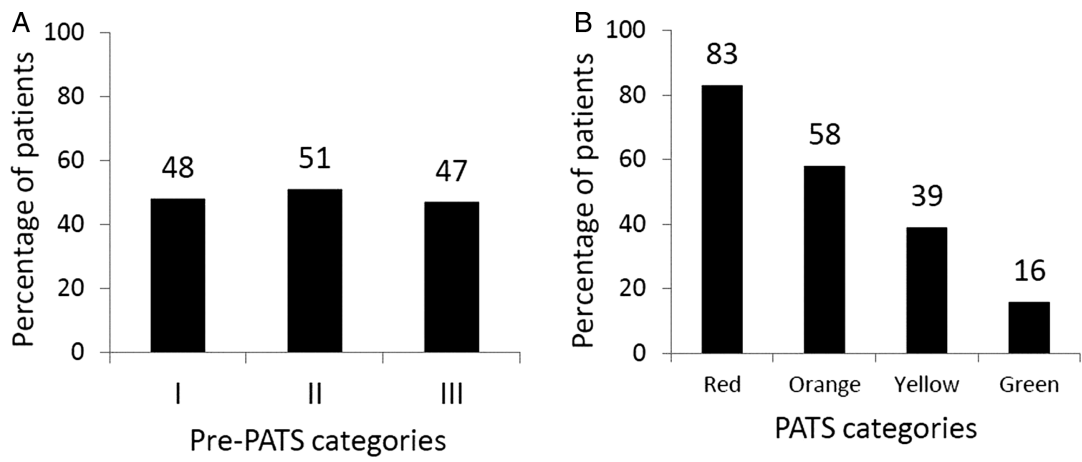

these patients in the PATS period were identified as needing urgent resuscitation and had the PATS process abbreviated without a triage-level assignment. Other reasons for nonassignment during PATS could be from an active decision not to use PATS (due to time constraints or a personal decision) or passive lack of knowledge regarding proper PATS methodology.

There were higher rates of admission and discharge in the PATS period. This may be because disposition was unknown for more patients in the pre-PATS period (12\%). If there was a true difference in illness severity between the two study periods, one might expect the changes in admission and discharge rates to be discordant (ie, one rate would increase while the other one would decrease). Differences between left without being seen rates and age groups between the pre-PATS and PATS periods may have been due to lack of reporting in the pre-PATS period.

\section{LIMITATIONS}

This study had several limitations. The patient data from both study periods were retrospectively entered into the database from the original medical records, resulting in missing or inaccurate data entry. As an example, 1792 (12\%) and 1205 (5\%) of the pre-PATS and PATS patients, respectively, did not have final dispositions. Similar rates for incomplete data have been reported in other triage studies, with ESI $^{9}$ and SATS $^{6}$ studies reporting $8 \%$ and $20 \%$, respectively. PATS likely had a higher rate of final disposition assignment due to an improvement in the triage form design. Despite the high proportion of patients with unknown dispositions in the pre-PATS group, the improvements in overtriage and undertriage rates with PATS were still significant. Even if every unknown pre-PATS-I patient $(n=25)$ was admitted and every unknown pre-PATS-III patient $(n=694)$ was discharged, the overtriage and undertriage rates would have improved only to $47 \%$ and $41 \%$, respectively, which would still be significant $(p<0.05)$. One related limitation is that we used admission as our primary endpoint as was done in the initial SATS study. ${ }^{6}$ Although it would have been preferable to also include resource utilisation as in the ESI triage studies, our triage forms did not reliably record this measure. ${ }^{9}$

Another limitation is that we have no record of the number of lost triage forms and the subsequent missing subjects from both study periods. We know we had no forms from 8 days in the PATS period (most of which were concentrated in July and August 2010), and our average number of recorded patients per day decreased by $22 \%$ in the PATS period. Anecdotally, there were never any days in the PATS period without patients, and the A\&E volume of patients remained relatively constant across study periods. Some of this decrease in average patients per day was therefore due to lost triage forms. However, given that the lost forms occurred mostly during an isolated time period, we do not believe that they would have had a significant impact on our results. It is also possible that the average number of patients seen per day could have decreased because a regional private hospital (Bokamoso) opened during the PATS study period.

Finally, this was a single-centre study using a before-and-after study design. An interrupted time series design might have been preferable from a research design standpoint, but it would have been technically difficult to implement with the dangerous potential for lost buy-in from nurse stakeholders who might have found the start-and-stop aspects of the intervention to be unacceptable. Future studies using PATS in other Botswana hospitals would increase the study's validity and the extent to which it can be generalised. Of note, the second largest Botswana hospital (Nyangabgwe) was impressed enough by PATS to implement it in their A\&E and outcomes are currently being studied.

\section{CONCLUSIONS}

Because of the resource-limited nature of medical care in $\mathrm{PMH}$, an effective triage system that sorts patients appropriately into various severity categories is necessary. Local adaptation of SATS into PATS generated a reliable and valid triage system, as
Figure 3 Proportion of critical patients (admitted to Intensive Care Unit or died in A\&E) in each triage category. (A) Proportion of critical patients $(n=79)$ within each pre-PATS category. (B). Proportion of critical patients $(n=63)$ within each PATS category (unk=unrecorded triage level). PATS, Princess Marina Hospital Accident and Emergency Triage Scale.
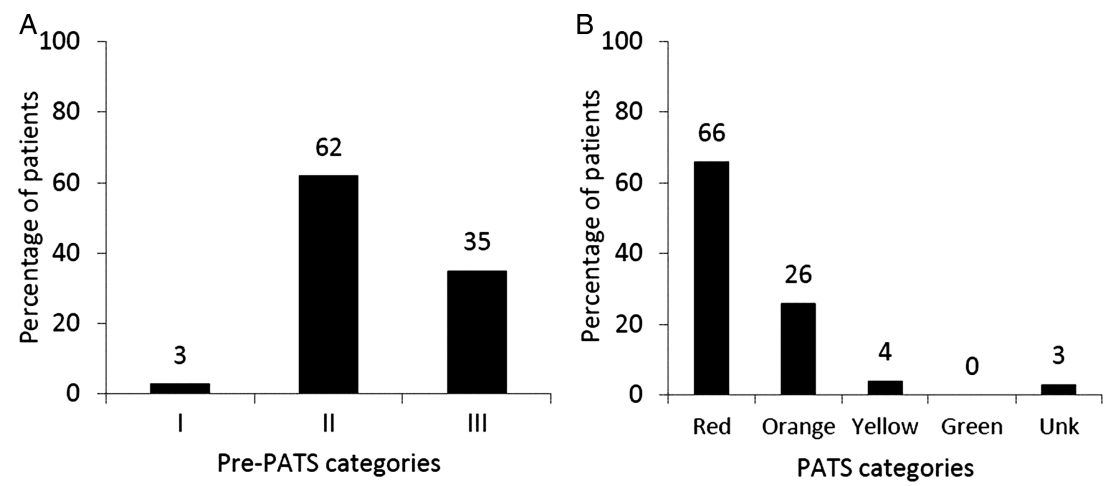
evidenced by a higher inter-rater reliability during training ${ }^{3}$ and improved predictability of patient severity when compared with the pre-PATS system. Future studies on patient flow (door to physician time, door to discharge time, etc), vital sign compliance, staff satisfaction and patient satisfaction could be conducted to determine some of the balance measures of this new system. Given the brief training requirements and objective methodology of the PATS system, it could be a sustainable triage scale in this resource-limited setting and could continue to be modified to meet the triage needs of a mixed adult and paediatric emergency department in other similar settings. The project implementation team is continuing to analyse data to track clinical outcomes, optimise resource utilisation, and minimise morbidity and mortality in the A\&E at PMH.

Acknowledgements PMH Staff, Kolobetso Malatsi, Beauty Sepelete, Ibraham Sani, Lefika Kareng, Jephres Muzondo, healthcare auxiliaries, Lee Ligon and Sartaj Alam.

Contributors PCM: conception and design of PATS; collection, analysis and interpretation of data; drafting of the article and incorporating coauthor feedback; final approval of the submission. SBT: conception of PATS; analysis and interpretation of data; revision of article for important intellectual content; final approval of the submission. AC and NC: implementation of PATS; collection, analysis and interpretation of data; revision of article for important intellectual content; final approval of the submission. AK: conception and design of PATS; collection and interpretation of data; drafting of the article and incorporating coauthor feedback; final approval of the submission.

Funding Programmatic costs: University of Botswana School of Medicine or PMH under Ministry of Health authority. Travel costs: Baylor College of Medicine.

\section{Competing interests None.}

Ethics approval Baylor College of Medicine, Botswana HRDC, University of Botswana and Princess Marina Hospital.
Provenance and peer review Not commissioned; externally peer reviewed.

Data sharing statement There exist additional data on triage timing, emergency department diagnoses and vital signs of patients who were subjects during the study period. These data are available with Dr Paul Mullan and Dr Amit Chandra in a secured, electronic format.

\section{REFERENCES}

1 Bateman C. New triage system halves mortalities. S Afr Med J 2006;96:770-2.

2 Hodkinson PW, Wallis LA. Emergency medicine in the developing world: a Delphi study. Acad Emerg Med 2010;17:765-74.

3 Twomey M, Mullan PC, Torrey SB, et al. The Princess Marina Hospital accident and emergency triage scale provides highly reliable triage acuity rating. Emerg Med J 2012 Aug;29:650-3.

4 Molyneux E, Ahmad S, Robertson A. Improved triage and emergency care for children reduces inpatient mortality in a resource-constrained setting. Bull World Health Organ 2006;84:314-19.

5 Gottschalk SB, Wood D, DeVries S, et al., Cape Triage Group. The Cape Triage Score: a new triage system South Africa. Proposal from the Cape Triage Group. Emerg Med J 2006;23:149-53.

6 Bruijns SR, Wallis LA, Burch VC. A prospective evaluation of the Cape triage score in the emergency department of an urban public hospital in South Africa. Emerg Med J 2008:25:398-402.

7 Emergency Medicine Society of South Africa-Practice Guideline EM 014Implementation of the South African Triage Scale. http://emssa.org.za/documents/ em014.pdf (accessed 6 Jun 2012).

8 Bruijns SR, Wallis LA, Burch VC. Effect of introduction of nurse triage on waiting times in a South African emergency department. Emerg Med J 2008;25:395-7.

9 Wuerz RC, Travers D, Gilboy N, et al. Implementation and refinement of the emergency severity index. Acad Emerg Med 2001;8:170-6.

10 Moll HA. Challenges in the validation of triage systems at emergency departments. J Clin Epidemiol 2010;63:384-8.

11 American College of Surgeons. Resources for the optimal care of the injured patient. American College of Surgeons, 1999.

12 Wuerz RC, Milne LW, Eitel DR, et al. Reliability and validity of a new five-level triage instrument. Acad Emerg Med 2000;7:236-42. 